\title{
Impact De L'utilisation Des Produits Phytosanitaires En Production Cotonniere Et Cacaoyere Sur La Production Apicole En Côte D'ivoire
}

\author{
Ehouman J B Ohoueu, DEA, et Doctorant, \\ Eboua N. Wandan, PhD, Maitre de Conférences, \\ Dofoungo Kone, Ingénieur et Doctorant, \\ Brou Aubin Assielou, DEA et Doctorant,
}

Institut National Polytechnique-Félix Houphouët Boigny de Yamoussoukro, UMRI Sciences agronomiques et Génie Rural, Laboratoire Sciences, Société, Laboratoire Environnement \& Gestion des Ressources Naturelles, BP 1313, Yamoussoukro

Dembélé Ardjouma, Dr, Maitre de Recherche, Laboratoire Agrochimie et Ecotoxicologie, LANADA, Abidjan, Cote d'Ivoire

\begin{abstract}
Beekeepers in northern and central part of Côte d'Ivoire, who are generally farmers, complain about desertions and frequent collapses of colonies. The application of pesticides to protect crops against attacks from disease and predators could be one of the factors responsible for these collapses and desertions. The objective of this study was to investigate if the use of agrochemicals in cotton and cocoa plantations could be a constraint to beekeeping. An interview implicating 51 beekeepers-farmers from eight cocoa and cotton producing regions was performed. The main results showed that $22 \%$ of the respondents were women. The apiaries contained 8 to 334 beehives with an average of 105 hives per apiary. Among a total of 5372 hives, 1402 were colonized which represents $26 \%$ of the hives.

Most of the pesticides: insecticides (50\%), herbicides (13\%) and fungicides $(9 \%)$ were used in cotton and cocoa production. Among farmers, $51 \%$ were not aware of the negative impact of agrochemicals usage on beekeeping. Moreover, $69 \%$ of them assured that they are over applying these chemicals. Besides that chemical applications were done during flowering period. From all these results, one can state that the use of agrochemicals on cotton and cocoa production might cause the desertions of bee, the weakening and collapse of hives that were observed by beekeepers.
\end{abstract}


Keywords: Agrochemical, beekeeping, agriculture, desertion, impact

\section{Résumé}

Les apiculteurs ivoiriens, qui sont pour la plupart des agriculteurs, se plaignent de fréquentes désertions et effondrements des colonies d'abeilles. L'utilisation des pesticides en agriculture pourrait être un des facteurs de ces phénomènes. L'objectif de cette étude était de vérifier si l'utilisation des produits agrochimiques en production cotonnière et en cacaoculture pourrait expliquer ces observations. Il s'est agi d'une enquête auprès de 51 agriculteurs-apiculteurs issus des régions du nord et du centre de la Cote d'Ivoire. Les résultats obtenus indiquent que $78 \%$ des apiculteurs sont de sexe masculin et qu'on compte 5372 ruches soit environ 7 ruches par enquêtés. Parmi les ruches 1402 sont colonisées, soit $26 \%$ des ruches en moyenne. Les produits phytosanitaires utilisés en cacaoculture et en culture cotonnière sont par ordre d'importance: les insecticides (50\%), les herbicides (13\%), les fongicides (9\%); et autres (28\%). Parmi les enquêtés, $51 \%$ ne savent que les produits phytosanitaires pourraient avoir un impact négatif sur la production apicole. En plus, $60 \%$ affirment utiliser ces produits à des doses au-dessus de celles préconisées. En plus de cela, les applications sont faites pendant la période de floraison des plantes mellifères; période d'activité intense de butinage des abeilles. Ces résultats montrent que l'application des produits phytosanitaires sur les cultures de cacao et de coton pourrait être la cause des désertions et effondrements observés par les apiculteurs de ces régions.

Mots clés: Produits agrochimiques, apiculture, agriculture, désertion, impact

\section{Introduction}

L'économie de la Côte d'Ivoire est basée sur l'agriculture, précisément sur les cultures pérennes telles que la cacaoculture, la cotonculture, etc. Afin d'augmenter leur production, les agriculteurs utilisent des produits phytosanitaires pour lutter contre les ravageurs et les maladies responsables de nombreux dégâts sur les cultures et les récoltes.

les abeilles butinant sur les cultures ayant reçu les applications de produits phytosanitaires ou sur les cultures environnantes peuvent entrer en contact avec ces produits or celle-ci sont source de pollinisation des plantes et garant de la biodiversité dans le monde (UNEP, 2010). En plus, leur élevage, l'apiculture est une pratique qui est de plus en plus exercée comme une activité complémentaire à l'agriculture ou comme activité d'appuis ou de divertissement par certaines populations (Rafalimanana, 2003). En Côte d'Ivoire, la pratique permet la production de miel, de la cire, du pollen et de 
la gelée royale. En 2008, le pays comptait environ 250 apiculteurs disposant 12.000 ruches et produisant 645 tonnes de miel naturel ; ce qui apportait un milliard de F CFA par an au PIB ivoirien (MEF, 2008).

Ces dernières années, on a régulièrement observé des phénomènes d'affaiblissement de ruchers avec une diminution de l'activité des abeilles sans que l'on ait pu observer la présence d'agents pathogènes Rafalimanana (2003). Les apiculteurs ont aussi évoqué la rareté et l'instabilité des colonies d'abeilles. L'objectif de cette étude est d'évaluer si l'utilisation des produits phytosanitaires dans les plantations de cacao et coton au nord et au centre de la Côte d'Ivoire est un frein à l'apiculture. Il s'est agi spécifiquement d'évaluer l'utilisation des produits phytosanitaires dans les zones de production de miel et de déterminer l'interaction entre l'utilisation des produits phytosanitaires en agriculture et les difficultés rencontrées en apiculture.

\section{Méthodologie \\ Sites d'étude}

L'étude a été menée dans le nord et le centre de la Côte d'Ivoire (Figure 1), zone de production agricole et apicole par excellence et forte utilisation de produit phytosanitaires (MEF, 2008). Dans chacune des régions considérées, des localités abritant de rucher ont été choisies.

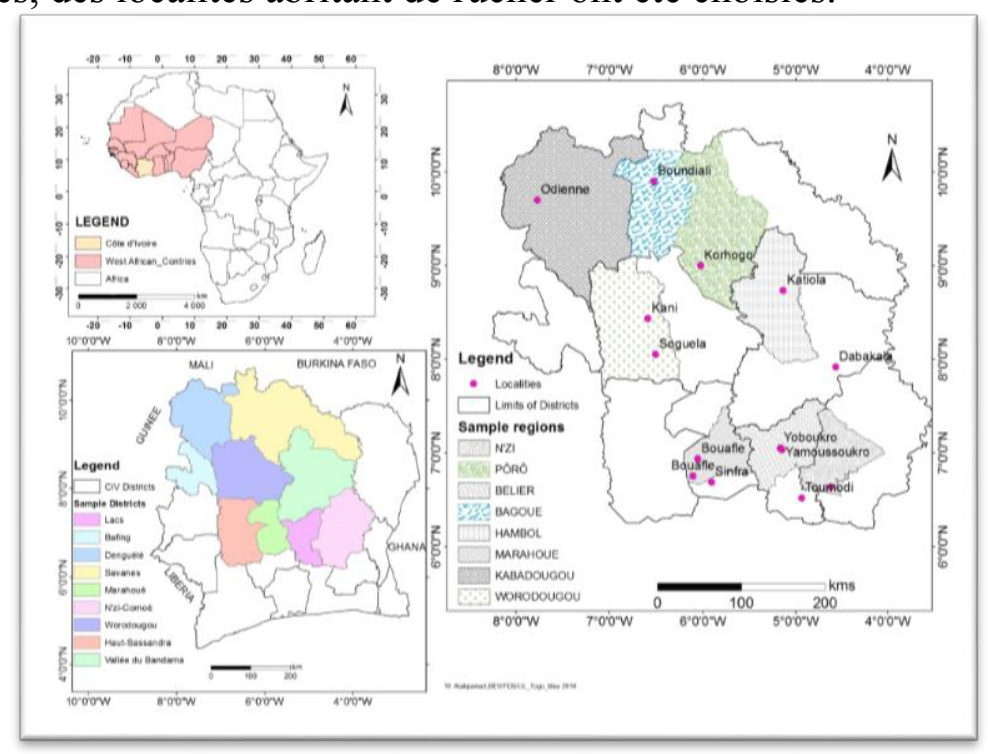

Figure 1: Localisation des régions d'étude au Centre et au Nord de la Côte d'Ivoire

\section{Collecte et traitement des données}

Les enquêtes se sont déroulées entre les mois de Janvier à Mars et Août 2015 durant la période de récolte de miels, les apiculteurs étant plus 
accessibles à cette période. La population cible était constituée d'individus pratiquant à la fois, l'apiculture (disposant au minimum de 3 ruches) et l'agriculture avec utilisation de produits phytosanitaires et dont les parcelles agricoles sont situées à au plus $3 \mathrm{~km}$ de rucher (Rafalimanana, 2003 ; Larroque, 2002). Sur cette base, 51 apiculteurs-agriculteurs ont été questionnés dont 35 au centre et 16 au nord de la Côte d'Ivoire.

Des entretiens individuels sur la base de questions ouvertes, indirectes et directes (Koudegnan, 2015; Touckia, 2015) testées au préalable sur trois apiculteurs/agriculteurs a permis de capter les informations sur la typologie des enquêtés, les caractéristiques de l'apiculture, la commercialisation des produits de la ruches, les activités agricoles, l'utilisation de produits phytosanitaires, et les périodes de floraison (Rafalimanana, 2003)

Les données obtenues ont été tabulées avec le logiciel Excel qui a servi à faire les graphiques. Les analyses factorielles de correspondances (AFC) ont été réalisées grâce au logiciel XLSTAT version 2008 sur les matrices de données contenant les variables éducation, type de produits phytosanitaires, avis sur les produits phytosanitaires, comportement des abeilles, les chutes, la colonisation et le dosage des produits phytosanitaires.

\section{Résultats}

\section{Caractéristiques de l'apiculture au Centre et au Nord de la Côte d'Ivoire}

A la fin de l'enquête, 51 apiculteurs ont été interviewés dans les huit régions dont 68,62\% au Centre et 31, 37\% au Nord (Fig. 2). Les jeunes apiculteurs ( $<30$ ans) ne représentent que $2 \%$ de l'ensemble contre $98 \%$ des adultes (30 à 60 ans). La moyenne d'âge des apiculteurs est de 42 ans. Les jeunes s'adonnent moins à l'apiculture car, ils ne perçoivent pas clairement le profit que l'apiculture pourrait leur apporter. Ce désintéressement des jeunes serait une menace pour la survie de l'apiculture dans ces zones. C'est une activité qui est majoritairement pratiquée par les hommes dans les deux zones d'études, les femmes rencontrées ne représentent que $31,5 \%$ des enquêtés. Ce taux des femmes est supérieur à ceux de Koudegnan et al (2015) où aucune apicultrice n'a été détectée au Togo. Il est quasi conforme à celui obtenu en Nouvelle-Calédonie qui est de $20 \%$ selon Larroque et al (2002). Cependant il est inférieur à ceux obtenus au Mozambique et à l'Ouganda selon Tchoumboue (2001). 


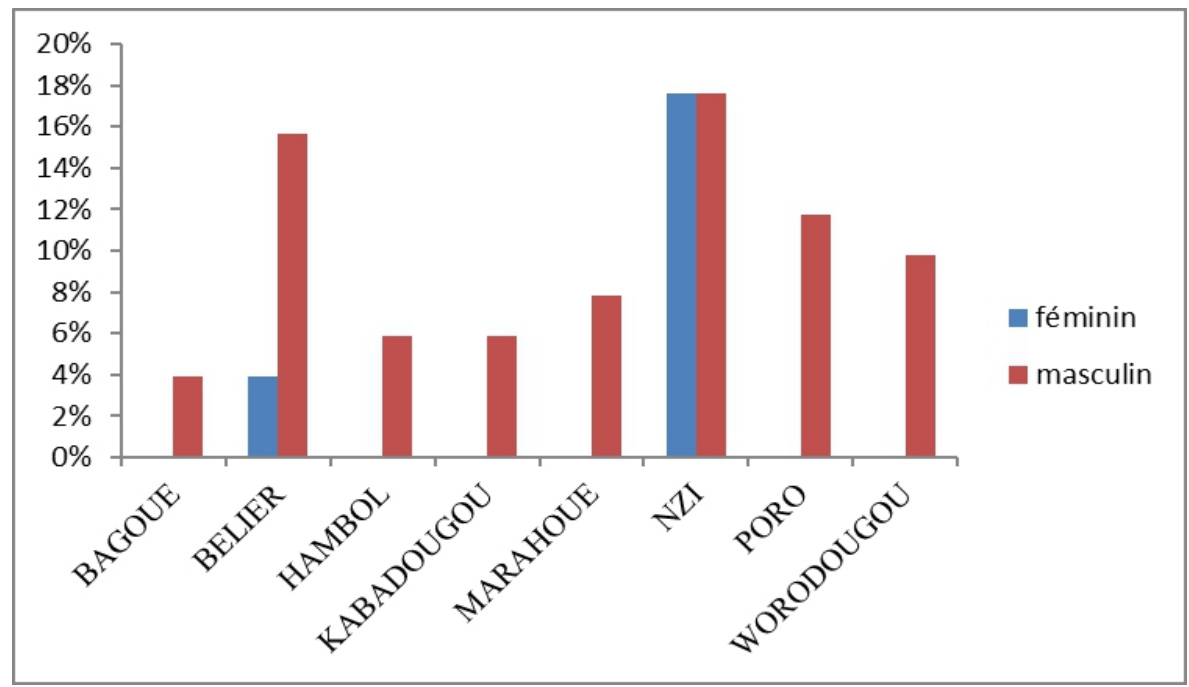

Figure 2: Sexe des apiculteurs/agriculteurs enquetés

En ce qui concerne le niveau d'éducation, on retient que $15,7 \%$ des apiculteurs/agriculteurs interrogés sont analphabètes, contrairement aux données recueillies par Koudegnan (2015) au Togo où tous les apiculteurs interrogés ont au moins le niveau du primaire.

La plupart des apiculteurs/agriculteurs enquêtés $(96,1 \%)$ appartiennent à une coopérative. Parmi eux, 70,6\% font partir à la fois d'une coopérative de production et de vente ; $21,6 \%$ font partie uniquement d'une coopérative de production et 3,9\% appartiennent à une coopérative de vente. Très peu $(3,9 \%)$ n'appartiennent à aucune coopérative.

Les apiculteurs/agriculteurs questionnés ont en moyenne 8 années d'activités apicoles et ont reçu en moyenne 2 formations au cours de leurs activités. Ces formations ont été financées par des projets $(72,5 \%)$ ou des ONG (23,5\%). Les formations ont assurées soit par des agents l'ANADER (51\%) ou par des consultants $(49 \%)$.

Les 51 apiculteurs-agriculteurs enquêtés disposent de 5372 ruches soit environ 7 ruches par enquêtés. Parmi les ruches 1402 sont colonisées, soit $26 \%$ des ruches. Les résultats indiquent qu'un bon nombre d'enquêtés (33\%) possèdent un nombre élevé de ruche (101-300), 27\% disposent d'un nombre moyen de ruche (21-40) alors que $21 \%$ ont un petit nombre de ruche (1-10). Ceux qui possèdent plus de 300 ruches ont peu (4\%). On constate par ailleurs que $33 \%$ de enquêtés ont 1 à 10 ruches colonisées. Ces résultats sont inférieurs à ceux obtenus au Cameroun où $43,3 \%$ des ruches étaient colonisées (Tchoumboué, 2001).

Deux types de ruches (Kenyane et Langsthroth) sont majoritairement utilisés au centre et au nord de la Côte d'Ivoire. La ruche Kenyane est la ruche la plus utilisée dans les deux zones (56,9 \%). Plusieurs apiculteurs 
expliquent le choix de ruche kenyane par son coût d'acquisition réduit $(18000 \mathrm{~F})$ par rapport à la ruche Lansgthroth $(24000 \mathrm{~F})$ et par la facilité de sa confection par les apiculteurs. Par ailleurs, les enquêtés indiquent que la ruche Kenyane est plus colonisée par les abeilles (51 \%) que la ruche Langsthroth $(31 \%)$. Cette préférence des abeilles pour la ruche kenyane serait due à sa forme horizontale qui lui donne l'allure d'une ruche naturelle. Bien que moins utilisée, la ruche Lansgtroth possède une capacité plu élevée en matière de capacité de stockage de miel (Gout et al, 2008).

Afin d'appâter les abeilles, de la cire fondue est mise dans les ruches qui sont par la suite déposées dans la nature. Dans le nord de la Côte d'Ivoire, $60 \%$ des apiculteurs installent les ruches sur les branches d'arbre pour faciliter la capture des abeilles; d'autres $(20 \%)$ par contre suspendent avec une corde les ruches aux arbres. Dans la zone centre du pays, $70 \%$ des apiculteurs déposent les ruches de capture sur des bidons remplis d'eau qui sont badigeonnés avec de l'huile de moteur usagée afin d'empêcher les fourmis d'avoir accès aux ruches. Parfois, les ruches badigeonnées sont déposée sur des briques (30\%).

Après la capture toutes les ruches colonisées sont rassemblées sur un espace nettoyé afin de constituer le rucher. Apres la constitution des ruchers, les récoltes sont effectuées dans $60 \%$ de cas, une fois par an, dans le 1er trimestre de l'année afin de réduire le taux de désertion. Cette même pratique a été observée au Cameroun (Tchoumboué, 2001) et au Togo (Koudegnan, 2015).

Les résultats indiquent par ailleurs que dans la zone centre, les $22 \%$ des enquêtés qui pratiquent une apiculture intensive, font deux récoltes de miel par an. On constate que même avec la pratique de l'apiculture intensive, on n'observe aucun élevage de reine de système de multiplication des abeilles alors que cette pratique permet d'avoir de bonnes et jeunes reines au moment voulu (Petit, 2002).

Tableau 1 : Quantité de produits de la ruche estimée par région

\begin{tabular}{cccc}
\hline \multirow{2}{*}{ Zone } & \multicolumn{3}{c}{ Quantité (tonnes) } \\
\cline { 2 - 4 } & Miel & Cire & Pollen \\
\hline Centre & $15,86 \mathrm{a}$ & $1,3 \mathrm{a}$ & $0,30 \mathrm{a}$ \\
Nord & $6,4 \mathrm{~b}$ & 1,106 & $0,02 \mathrm{~b}$ \\
Total & 23,143 & 2,406 & 0,32 \\
\hline
\end{tabular}

Trois produits ont été identifiés dans les deux zones de production ; il s'agit du miel, de la cire et du pollen. La production totale de miel a été estimée à 23,14 tonnes avec le centre ayant une production significativement plus forte (15,9 tonnes) par rapport au nord (6,4 T). Dans ces deux zones, le miel est commercialisé en moyenne, à $2000 \mathrm{~F}$ le kilogramme; ce prix de 
vente est supérieur à celui pratiqué en Tanzanie (Mbétid-Bessane, 2004) qui était de $500 \mathrm{~F} \mathrm{CFA} / \mathrm{kg}$.

En considérant qu'un apiculteur récolte en moyenne $453,78 \mathrm{Kg}$ de miel par an, il obtient un revenu brut annuel de de $907560 \mathrm{~F}$ soit $75630 \mathrm{~F}$ par mois. Ce revenu mensuel est supérieur celui des apiculteurs du Cameroun qui est de 42000 F (Tchoumboue, 2001). Concernant la cire, la production moyenne par apiculteur a été estimée à $48,18 \mathrm{~kg}$; vendue à $1300 \mathrm{~F}$ le Kg elle procure $62600 \mathrm{~F} /$ an soit $5219 \mathrm{~F}$ par mois. En considérant la production de pollen marginale, le chiffre d'affaire annuel moyen d'un apiculteur est estimé à $969160 \mathrm{~F}$. En considérant les charges d'exploitation à $51 \%$ du CA et l'amortissement d'une ruche à 3150 F CFA (Mbétid-Bessane, 2004 ; Matsop et al 2011), le revenu annuel net d'un enquêté et estimé à 470 575 F CFA.

\section{Perception des conséquences de l'utilisation des produits phytosanitaires par les apiculteurs/agriculteurs}

Dans les régions du nord de la Côte d'Ivoire, les résultats indiquent que les enquêtés cultivent dans 31\% de cas, du cotonnier (Gossypium arboreum) et du manguier (Manguifera indica) et dans $19 \%$ de cas de l'anacardier (Anacardium occidentale) et des céréales. Au centre par contre, on observe une diversification de la production agricole: le cacao (Theobrama cacao) (34\%), le café (coffea rabica) $(20 \%)$, les citrus (14\%), les Poaceae (11\%), et le manguier (Manguifera indica) (9\%) (Figure 3). La majorité des cultures reçoivent des produits phytosanitaires.

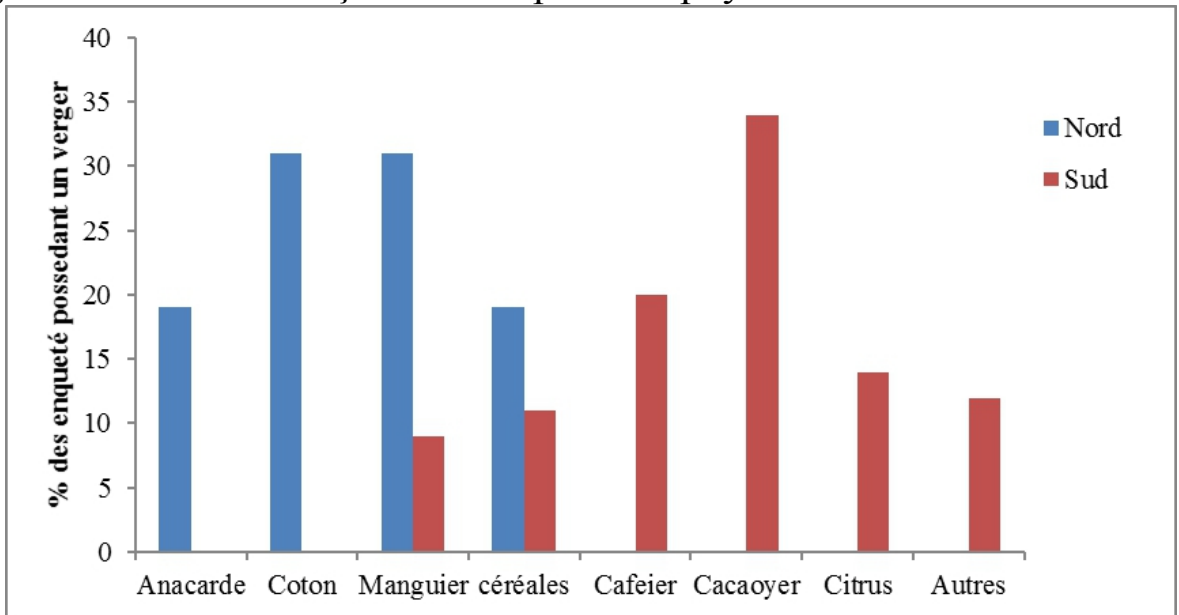

Figure 3: spéculations pérennes pratiquées par le enquêtés dans le zone nord et sud de la Côte d'Ivoire

Plusieurs produits phytosanitaires sont appliqués sur les cultures dans les deux zone, à savoir les insecticides (50\%), les herbicides (13\%), les fongicides $(9 \%)$ ou le trois à la fois $(28 \%)$ (Figure 4$)$. 


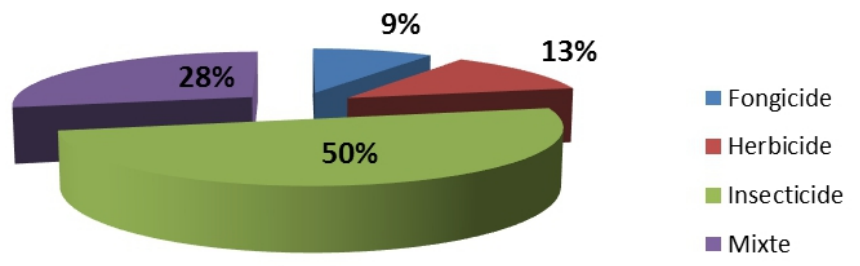

Figure 4: Fréquence de types de produits phytosanitaires utilisés dans les zones d'étude

La fréquence d'utilisation et fonction de la région; dans la région du Bélier (centre), $25 \%$ de enquêtés affirment appliquer tous les types de produits alors que dans la région du Pôrô, $30 \%$ des produits sont des insecticides. Ceci s'explique par le fait que la culture dominante, le coton requiert une lutte contre les insectes qui s'attaquent à la fibre, dépréciant ainsi sa qualité.

Environ la moitié (49\%) des apiculteurs/agriculteurs enquêtés indique que le produits phytosanitaires ont néfastes et 35\% affirment ignorer les impacts négatifs potentiels des produits phytosanitaires sur leurs activités, eux-mêmes et sur leur environnement. En fait, ces thèmes n'ont pas été abordés au cours des formations qu'ils ont reçues.

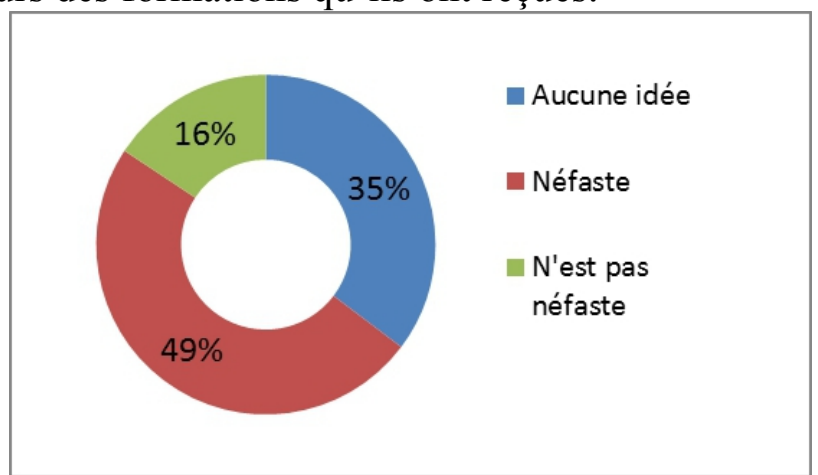

Figure : Perception des apiculteurs concernant les conséquences de l'utilisation des produits phytosanitaires

En termes d'utilisation de produits phytosanitaires, tous les enquêtés dans le nord font 6 applications d'insecticides par saison de culture de coton. Au centre $51 \%$ des enquêtés appliquent deux fois par an des fongicides et des insecticides sur le verger de cacao. La majorité des enquêtés (69\%) 
estiment qu'ils font du surdosage lors des préparations des bouillies pour l'épandage. Ils expliquent ce comportement par le fait que plus de produit appliqué entrainerait la destruction totale des ravageurs, ce qui garantirait une bonne récolte. Ces mêmes observations ont été faites sur les agrumes à Madagascar (Rafalimanana, 2003).

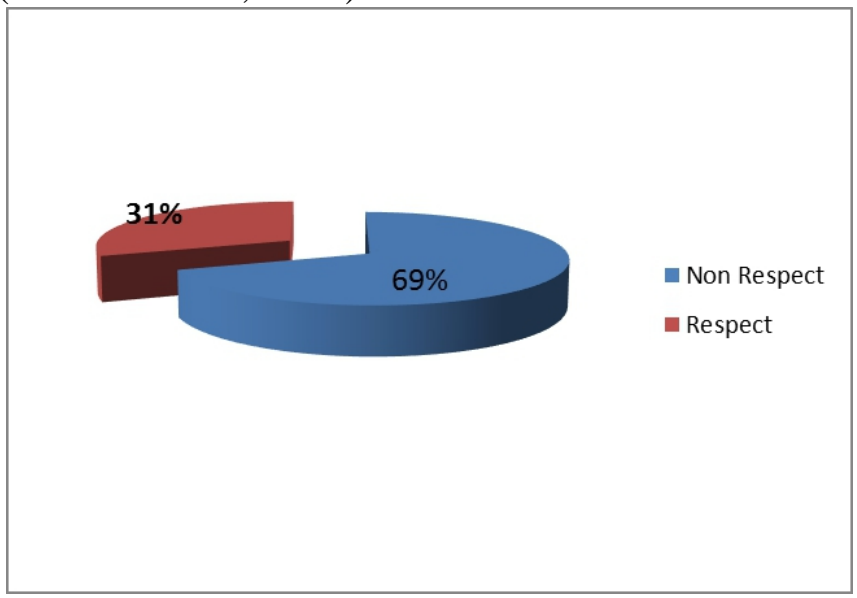

Figure:Fréquence d'utilisation des produits phytosanitaires

Le cotonnier fleurit durant 3 à 4 mois dans l'année (Juillet - Octobre) et c'est au cours de cette période que se font les applications de produits phytosanitaires. Concernant le cacao, la floraison est quasi permanente mais les traitements phytosanitaires interviennent en décembre et en juillet. Dans les agrumes, les pulvérisations de pesticides ont lieu pendant les périodes de floraison pour lutter contre les mouches des fruits (Delorme, 2002; Rafalimanana, 2003). Comme on le constate, pour toutes ces spéculations, les périodes de traitement phytosanitaires coïncident avec la période de butinage des abeilles. Ce qui met en danger la survie des abeilles qui sont en fait des populations non cible de ces produits. C'est pour palier cela, que dès 1975, une loi prise en France interdit l'usage des insecticides et acaricides pendant la période de floraison et de production du miellat. 


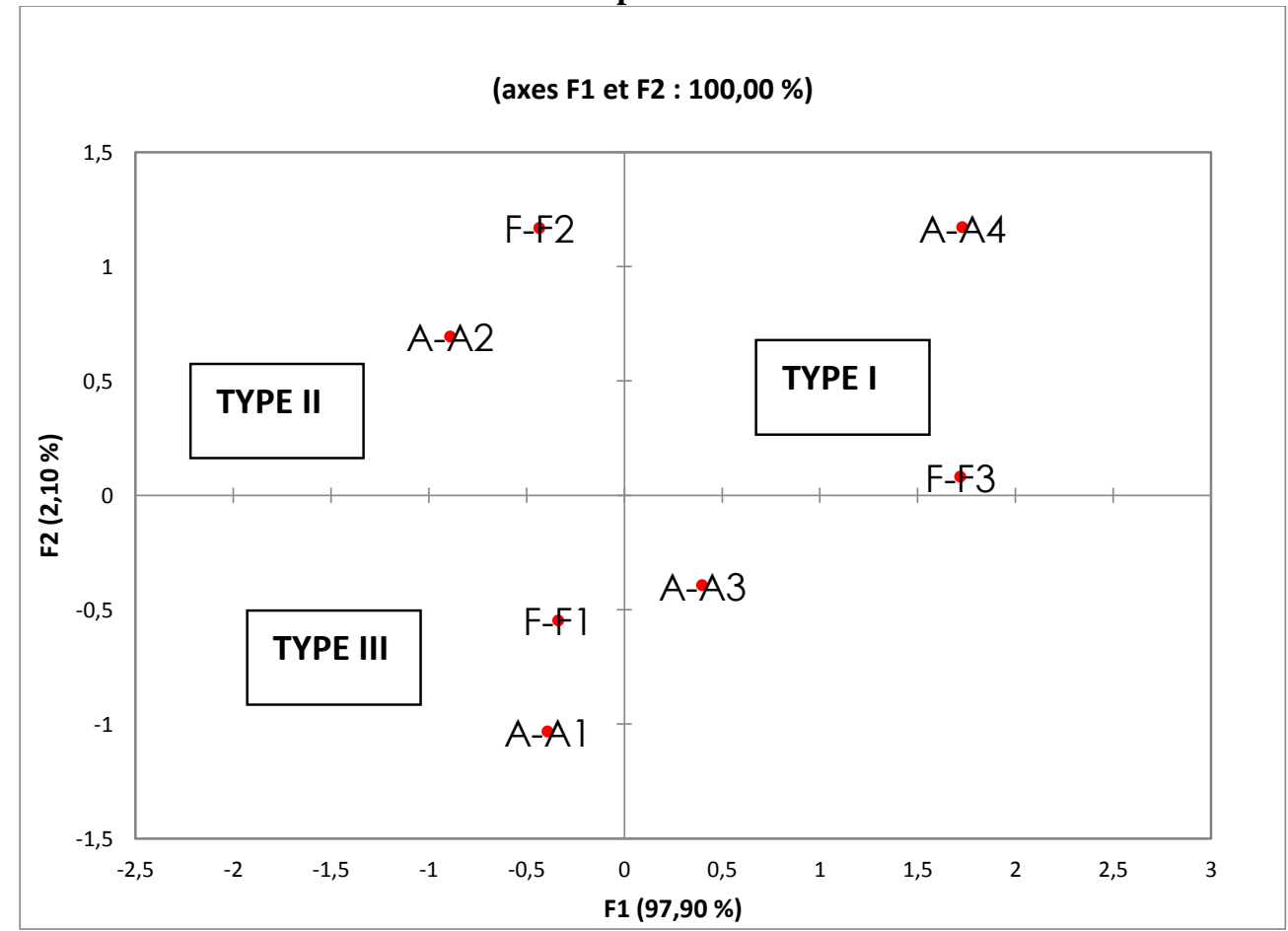

Figure 5: Liens entres les niveaux d'éducation et la colonisation des ruches

A : Education, A1 : Analphabète, A2 : Primaire, A3 : Secondaire, A4 : Supérieure, F :

Colonisation

F1 : Faible colonisation (1-10), F2 : Colonisation moyenne $(11-70)$, F3 : Forte colonisation $(71-300)$

Quand on regarde la relation entre le niveau d'éducation des apiculteur/agriculteurs, on voit que les deux premiers axes F1 et F2 de l'AFC (Figure 5) expliquent la totalité de la variabilité globale. Le premier axe F1 contribue à $97,90 \%$ et le second à $2,10 \%$. On constate donc qu'il y a une forte corrélation entre le niveau d'instruction de l'apiculteur/agriculteur et le niveau de colonisation des ruches. En effet, les apiculteurs qui ont fait des études supérieures ont des ruches avec de fortes colonisations (71 à 300 ruches colonisées) alors que ceux qui ont le niveau d'étude plus faible (primaire) possèdent des ruches avec une colonisation moyenne (11 et 70 ruches) (type II). Ceux qui sont analphabètes ont des ruches à faible colonisation (1 à 10 ruches). Ces résultats s'expliqueraient par le fait que ceux qui ont un niveau d'instruction élevé auraient une meilleure conduite de leurs élevages et de bonnes pratiques agricoles qui favoriseraient une bonne colonisation des ruches.

En ce qui concerne le comportement des abeilles en fonction du type de produits phytosanitaires utilisés dans les cultures, les deux premiers axes 
F1 et F2 de 1'AFC (Figure 6) expliquent $74,57 \%$ de la variabilité globale. Le premier axe $\mathrm{F} 1$ contribue à $60,48 \%$ et le second à $14,09 \%$. Ces résultats indiquent que l'utilisation des insecticides dans les cultures induirait un comportement anormal et la chute des abeilles. A l'inverse, le comportement normal des abeilles serait lié à la non-utilisation d'insecticides dans les cultures. Ce résultat sur le comportement anormal des abeilles vis à vis des produits phytosanitaires est conforme celui obtenu par Albouy (2014). L'utilisation de pesticides induirait un changement de comportement des abeilles tout en diminuant de $20 \%$ à $30 \%$ les colonies d'abeilles (Védrenne, 2002).

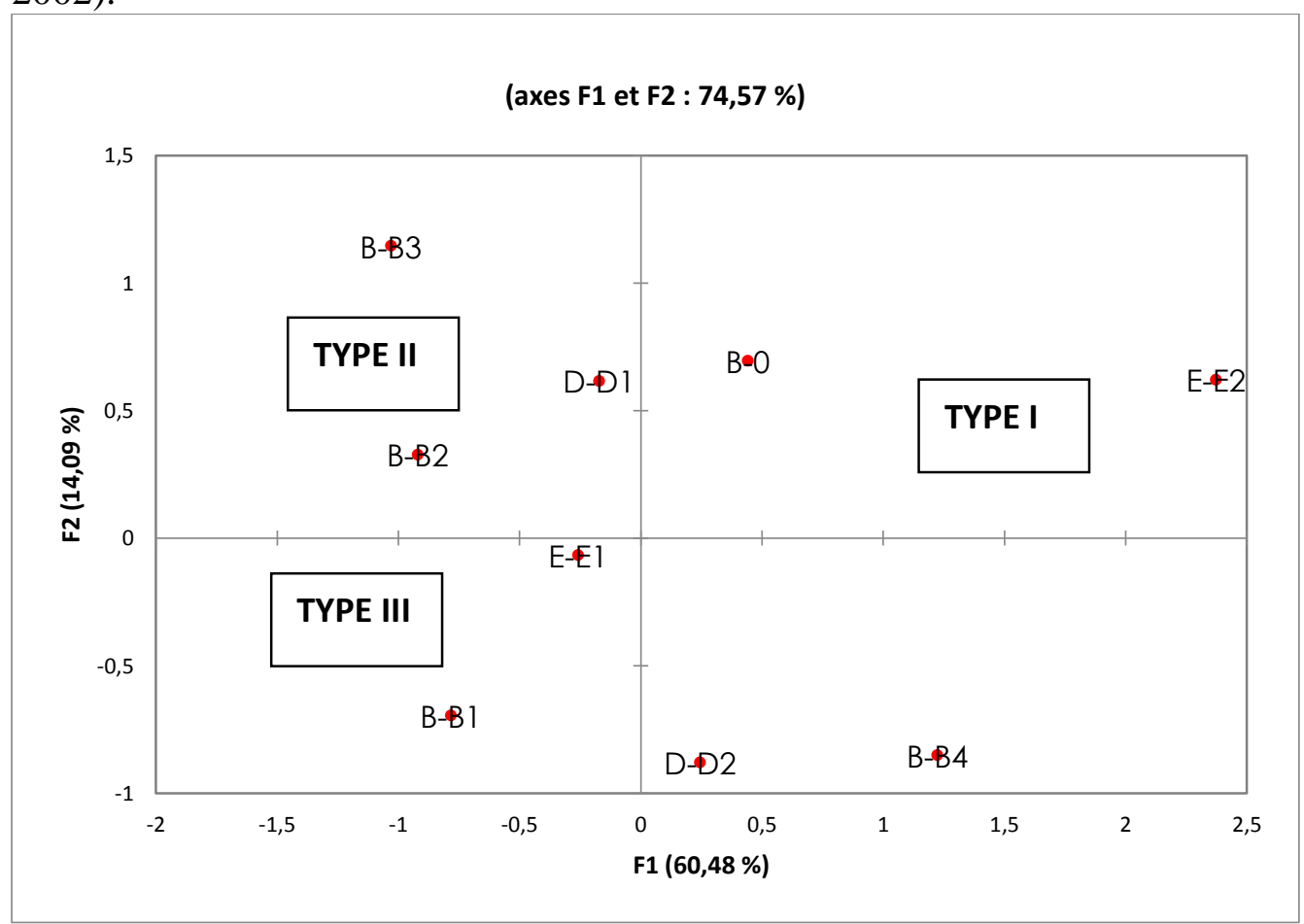

Figure 6: Relation entre le type de produits phytosanitaires utilisés en agriculture et le comportement d'abeilles

B : Type de produits phytosanitaires, B1 : Insecticide, B2 : Fongicide, B3 : Herbicide, B4 : Mixte (plusieurs produits phytosanitaires), D : Chute ou pas des abeilles, D1 : Chute, D2 :

Pas de chute, E : comportement des abeilles, E1 : Anormal, E2 : Normal

Quand on considère le comportement des abeilles en fonction du dosage des produits phytosanitaires, on constate que les deux premiers axes $\mathrm{F} 1$ et $\mathrm{F} 2$ de l'AFC (Figure 7) expliquent $81,50 \%$ de la variabilité globale. Le premier axe F1 contribue à $79,66 \%$ et le second à $1,84 \%$. On remarque que les chutes de colonie et le comportement anormal des abeilles serait lié au surdosage des produits phytosanitaires. Toute chose étant égale par ailleurs, 
le respect du dosage des produits phytosanitaires induit un comportement normal des abeilles. Ces résultats sont conformément ceux d'Anger (2009).

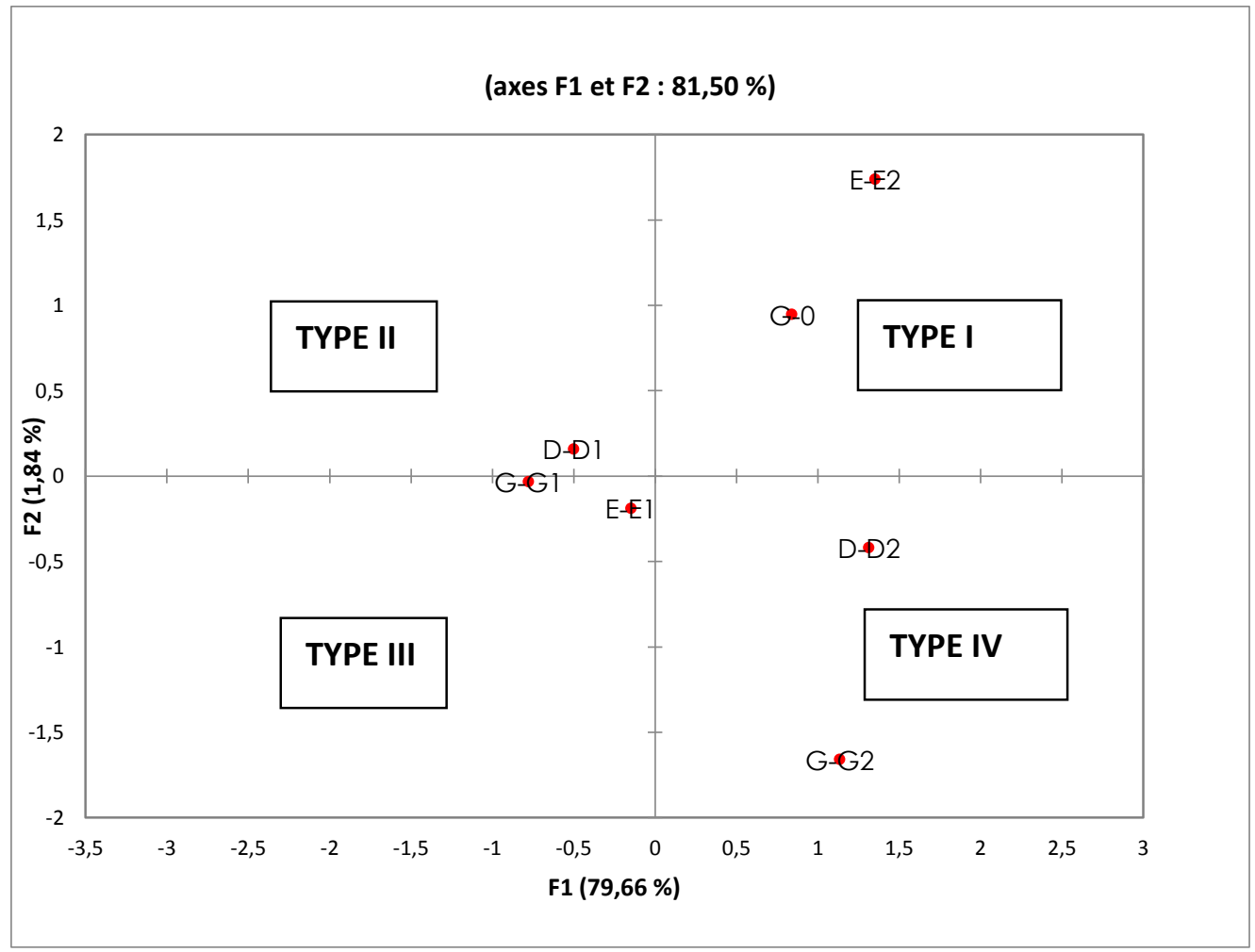

Figure 7: Lien entre le dosage des produits phytosanitaires utilisés dans les cultures et Comportement des abeilles

C1 : Néfaste, C2 : Pas néfaste, C3 : Aucune idée, D : Chute ou pas des abeilles, D1 : Chute, D2 : Pas de chute, E : comportement des abeilles, E1 : Anormal, E2 : Normal

\section{Conclusion}

L'apiculture est majoritairement une activité secondaire dans les deux zones enquêtées. La pratique de l'apiculture reste extensive, même si dans la zone centre on observe une apiculture intensive pratiquée par une minorité qui possède des équipements modernes.

Ces artisans, à la foi apiculteurs et agriculteurs, utilisent plusieurs produits phytosanitaires pour protéger leurs cultures contre les ravageurs et les maladies. Parmi ces produits, les insecticides sont plus utilisés au nord de la Côte d'Ivoire à causes de la culture de coton, principales culture de rente en zone de savane. Au Centre, du fait de l'humidité qui entraine le développement de maladies cryptogamiques et du fait aussi de la raréfaction de la main-d'œuvre, les insecticides sont aussi bien utilisés que le herbicides et les fongicides. Tous les acteurs s'accordent à dire qu'ils font du surdosage des produits utilisés. 
Bien qu'étant à la foi apiculteurs et agriculteurs, les personnes enquêtées ignorent complètement que l'utilisation des produits phytosanitaires peut avoir un effet délétère sur l'apiculture et leur santé. Les désertions, les effondrements et les affaiblissements des ruches observés par les apiculteurs peuvent s'expliquer par l'utilisation des produits phytosanitaires en agriculture pendant le temps de floraison des différentes cultures mellifères. D'autres études doivent être effectuées, comme l'évaluation des résidus chimiques sur les abeilles et les produits de la ruche afin de confirmer l'impact total de l'utilisation de ces produits sur l'activité apicole.

\section{References:}

1. Albouy V, Le Conte Y., 2014. Nos abeilles en péril. Éditions Quae. 192.

2. Anger J. P, Kintz P., 2009. Difficultés analytiques de la caractérisation des pesticides

3. Delorme R., Leroux P. \& Gaillardon P., 2002. Evolution des produits phytosanitaires à usage agricole. Les insecticides-acaricides. Phytoma. 548. 7-13.

4. Goût J., 2008. 250 réponses aux questions d'un ami des abeilles, éd. Le gerfaut, France. p. 143,

5. Koudegnan C, Nenonene A, Guelly K, Edorh T., 2015. L'apiculture dans la lutte contre les changements climatiques dans la zone écologique IV du Togo. Afrique science 11(6) (2015) 45 - 59

6. Larroque G, RATIA G., 2002. Audit du rucher calédonien, 69p.

7. Tsafack Matsop A. S., Muluh Achu G., Kamajou F., Ingram V. et Vabi Boboh M., 2011. Etude comparative de la rentabilité de deux types d'apiculture au nord-ouest Cameroun. Tropicultura, 29(1) : 3-7.

8. Mbétid-Bessane E. 2004. Apiculture, source de diversification de revenus des petits agriculteurs : cas du bassin cotonnier en Centrafrique. Tropicultura, 22(3) : 156-158.

9. Ministère de 1'Economie et des Finances. 2008. Rapport National d'Investissement de la Côte d'Ivoire. Conférence de haut niveau sur : L'eau pour l'agriculture et l'énergie en Afrique : les défis du changement climatique. Syrte, Jamhiriya Arabe Libyenne, 15-17. $12 \mathrm{p}$.

10. Petit D., 2002. Principes et méthodes d'élevage de Reines d'abeilles. http://daniel.petit.chez-alice.fr/index.htm,

11. Rafalimanana H. J., 2003. Evaluation des effets d'insecticides sur deux types d'Hyménoptères auxiliaires des cultures, l'abeille domestique (Apis mellifera L.) et des parasitoïdes de pucerons. Thèse unique, $17 \mathrm{p}$. 
12. TchoumbouéJ., Tchouamoà I.R., Pinta J.Y. et Nja M.N. 2001. Caractéristiques socio-économiques et techniques de l'apiculture dans les hautes terres de l'ouest du Cameroun. Tropicultura, 19(3) : 141-146. Touckia G. I, Yongo O. D, Etse K. D, Nzambo S, Gue Mamadou S, Kokou K., 2015. Évaluation des performances de Jatropha curcas L. dans les conditions pédoclimatiques du village de Nzila en République Centrafricaine (RCA). Afrique science, 11 (6): 181318548

13. UNEP, 2010. Emerging issues: Global Bee Colony Disorder and Other Threats to Insect Pollinisators.

14. Védrenne Y., 2002. L'apiculture en France. Le Courrier de la Nature. 196. 39-41. 